\title{
Temperature and Size Dependence of the Optical Properties of Tetrapod-Shaped Colloidal Nanocrystals Exhibiting Type-II Transitions
}

Giovanni Morello, ${ }^{*+}$ Angela Fiore, ${ }^{\dagger}$ Rosanna Mastria, ${ }^{\dagger}$ Andrea Falqui, ${ }^{\ddagger}$ Alessandro Genovese, ${ }^{\neq}$ Arianna Creti, ${ }^{\S}$ Mauro Lomascolo, ${ }^{\S}$ Isabella R. Franchini, ${ }^{\neq}$Liberato Manna, ${ }^{\ddagger}$ Fabio Della Sala, ${ }^{\dagger, \|}$ Roberto Cingolani, $^{\neq}$and Milena De Giorgi ${ }^{\dagger}$

${ }^{\dagger}$ Nanoscience Institute-CNR, Via per Arnesano, 73100 Lecce, Italy

${ }^{\ddagger}$ Istituto Italiano di Tecnologia, Via Morego 30, 16163 Genova, Italy

${ }^{\S} \mathrm{IMM}$ CNR, Institute for Microelectronic and Microsystems, I-73100 Lecce, Italy

"Center for Biomolecular Nanotechnologies @UNILE, Istituto Italiano di Tecnologia Via Barsanti, 73010 Arnesano (Lecce), Italy

ABSTRACT: We have investigated the optical properties of colloidal seed-grown CdSe (seed)/CdTe (arms) nanotetrapods both experimentally and computationally. The tetrapods exhibit a type-II transition arising from electrons localized in the CdSe seed region and holes delocalized in the CdTe arms, along with a residual type-I recombination in long-arm tetrapods. Experiments and theory helped to identify the origin of both types of transitions and their size dependence. In particular, timeresolved experiments performed at $10 \mathrm{~K}$ evidenced a sizedependent, long living type-II radiative emission arising from the peculiar electron-hole wave function localization. Temperature-dependent photoluminescence (PL) studies indicate that, at high temperature $(>150 \mathrm{~K})$, the main process limiting the PL quantum efficiency of the type-I PL is thermal escape of the charge carriers through efficient exciton-optical phonon coupling. The type-II PL instead is limited both by thermal escape and by the promotion of electrons from the conduction band of the seed region to that of the arms, occurring at $T>200 \mathrm{~K}$.

\section{INTRODUCTION}

Colloidal semiconductor nanocrystals have recently emerged as potential candidates for a wide range of applications, including lightemitting diodes (LEDs), ${ }^{1,2}$ lasers, ${ }^{3-5}$ solar cells, ${ }^{6,7}$ and multiplexing analysis in biology. ${ }^{8,9}$ Remarkable advances in synthetic approaches have enabled the fabrication of nanocrystals with both controlled shape and chemical composition. Semiconductor nanocrystals with rod shapes, for example, exhibit optical properties that are markedly different from those of spheres ${ }^{10-12}$ and that arise from the different type of quantum confinement occurring in rods as compared to spherical nanocrystals. ${ }^{10}$ Among the peculiar properties of nanorods, we mention one-dimensional exciton dynamics, high photoluminescence quantum yield (PLQY) at room temperature, linearly polarized emission, and large Stokes shift. ${ }^{11,13,64}$ Recently, the possibility to accomplish wave function engineering and exciton storage in nanorods was demonstrated, ${ }^{14,15}$ and new methods have been developed to assemble semiconductor nanorods with narrow distributions of lengths and diameters over large areas (up to several square millimeters). ${ }^{16-18}$

In contrast, tetrapod-shaped semiconductor nanocrystals are nanostructures in which four rod-shaped arms branch out from a central region according to a tetrahedral geometry. Their optical properties are similar to those of nanorods, except for the absence of polarization anisotropy in emission and absorption,

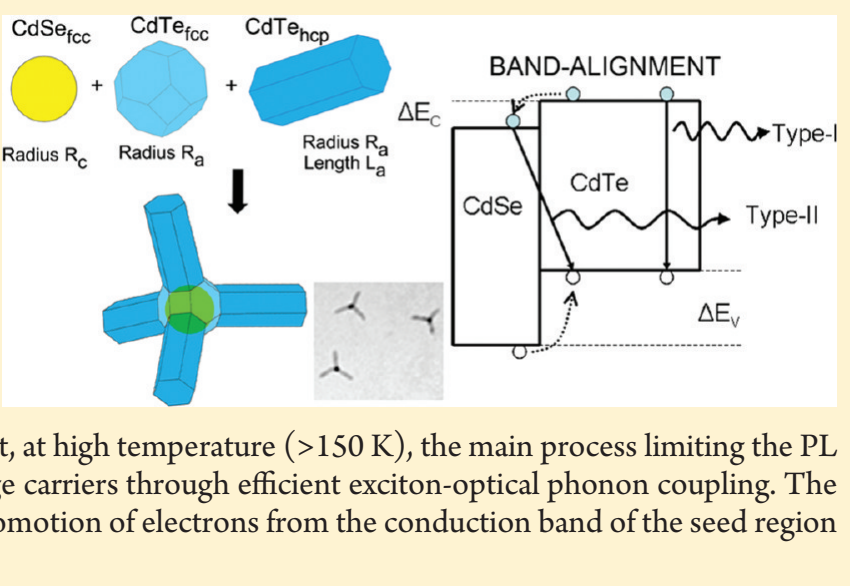

because of their tetrahedral symmetry. They too have attracted considerable interest in the past few years, because of their optical, ${ }^{55}$ electronic, ${ }^{19-25}$ and mechanical properties, ${ }^{26}$ as well as chemical reactivity. ${ }^{27,28}$ Also, they have been proposed for applications in different classes of devices, such as photovoltaic cells, ${ }^{6,29,30}$ single-nanoparticle transistors, ${ }^{31}$ electromechanical devices, ${ }^{26}$ and recently also scanning probe microscopes. ${ }^{32}$

In addition to shape control, it is now possible to synthesize, by colloidal approaches, nanoheterostructures consisting of sections of two different semiconductors, so that the relative band alignment at the interface between the two semiconductor regions can be of either type-I or type-II. The type-II heterostructure is particularly interesting, ${ }^{33-38,57,58,75}$ as electrons are localized in one semiconductor region and holes in another region, with the localization occurring at a short time after their generation (on the order of $500 \mathrm{fs}$ ). ${ }^{36}$ From these regions, carriers can recombine by emitting photons at energies lower than the respective energy gaps of the two semiconductor parts. Type-II materials are indeed ideal for photocatalytic

\footnotetext{
Received: $\quad$ May 24, 2011

Revised: July 21, 2011

Published: August 02, 2011
} 
Table 1. List of the Main Parameters of All the Samples of Tetrapods Studied: Nominal Diameter of Starting Seeds and Length of the Arms, Corresponding to the Maximum Value Extracted from TEM Observations; Energies of the Type-I $\left(E_{\mathrm{I}}\right)$ and Type-II $\left(E_{\mathrm{II}}\right)$ Transitions and Relative Difference $\left(\Delta E_{\mathrm{I}-\mathrm{II}}\right)$, Corresponding to the Actual Conduction Band Offset; Activation Energy of the Process Activated at Lower Temperature $\left(E_{\mathrm{a}}\right)$; Energy Spacing of the Two Lowest Absorption Peaks of the Ultimate Tetrapods $\left(\Delta E_{1,2}\right)$; Best-Fit Parameters $\left(E_{\mathrm{esc}}\right.$ and $\left.E_{\mathrm{b}}\right)$ Obtained from the PL Intensity Analysis, Representing the Escape Energy and the Energy of the Conduction Band Barrier, Respectively

\begin{tabular}{cccccccccc} 
sample & $\begin{array}{c}\text { CdSe seed } \\
\text { diameter }(\mathrm{nm})\end{array}$ & $\begin{array}{l}\text { CdTe arm } \\
\text { length }(\mathrm{nm})\end{array}$ & \multicolumn{1}{c}{$E_{\mathrm{I}}(\mathrm{eV})$} & $E_{\mathrm{II}}(\mathrm{eV})$ & $\Delta E_{\mathrm{I}-\mathrm{II}}(\mathrm{meV})$ & $E_{\mathrm{a}}(\mathrm{meV})$ & $\Delta E_{1,2}(\mathrm{meV})$ & $E_{\mathrm{esc}}(\mathrm{meV})$ & $E_{\mathrm{b}}(\mathrm{meV})$ \\
A1 & 3.7 & $6 \pm 1$ & $\mathrm{n} / \mathrm{a}$ & $1.223 \pm 0.001$ & $\mathrm{n} / \mathrm{a}$ & $\mathrm{n} / \mathrm{a}$ & $\sim 48$ & $59 \pm 1$ & $418 \pm 15$ \\
A2 & 3.7 & $9 \pm 1$ & $1.690 \pm 0.001$ & $1.272 \pm 0.001$ & $418 \pm 2$ & $\mathrm{n} / \mathrm{a}$ & $51 \pm 2$ & $\mathrm{n} / \mathrm{a}$ & $390 \pm 5$ \\
A3 & 3.7 & $14 \pm 1$ & $1.721 \pm 0.001$ & $1.272 \pm 0.002$ & $449 \pm 3$ & $9.7 \pm 1$ & $55 \pm 2$ & $58 \pm 1$ & $449 \pm 6$ \\
A4 & 3.7 & $25 \pm 1$ & $1.785 \pm 0.001$ & $1.317 \pm 0.001$ & $468 \pm 2$ & $13.9 \pm 2$ & $74 \pm 2$ & $70 \pm 1$ & $460 \pm 10$ \\
\hline
\end{tabular}

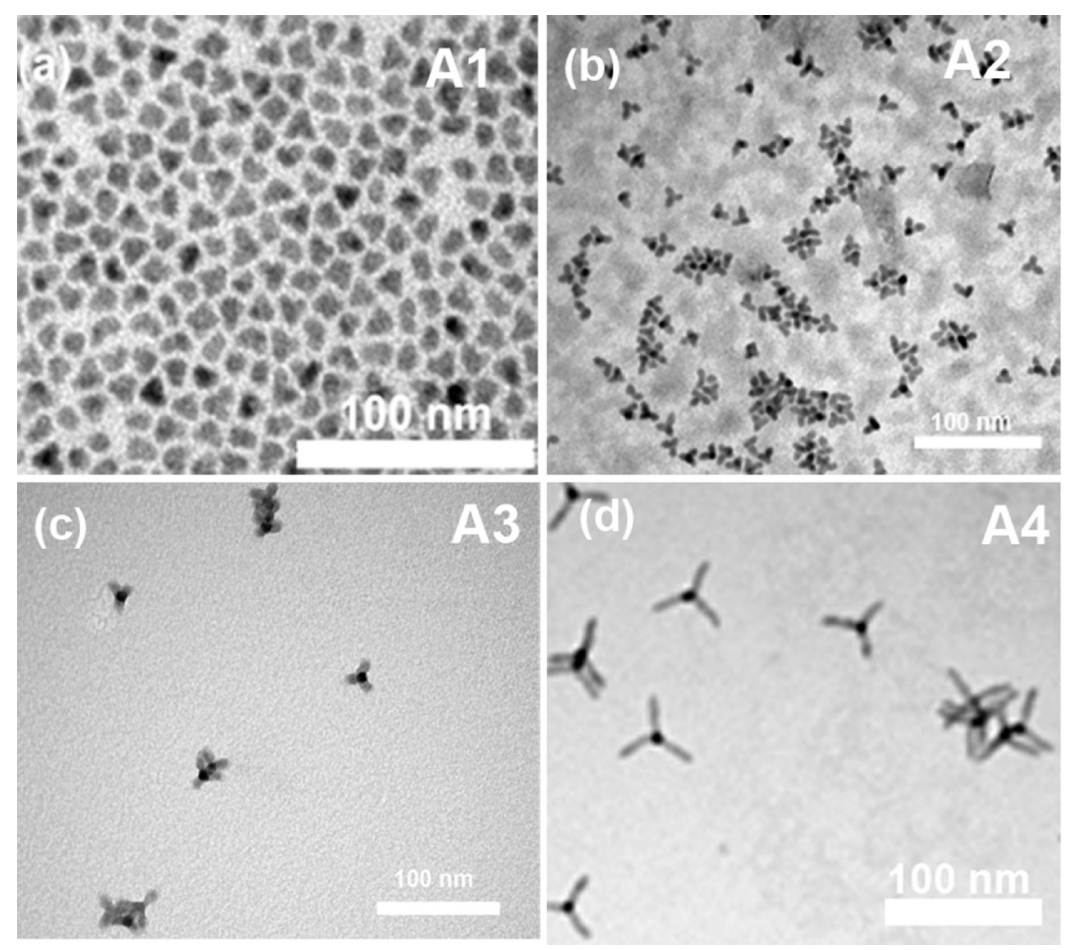

Figure 1. Low-magnification TEM images of the four samples of CdSe (core)/CdTe (arms) tetrapods, prepared starting from the same CdSe seeds and differing from each other in average arm length and diameter.

reactions $^{39,40}$ and for photovoltaics, ${ }^{10,41-43,74}$ in which both rapid charge separation and low radiative recombination efficiency are required. Recent developments in the synthesis of anisotropic heterostructures have involved rod-shaped nanoparticles consisting of a spherical semiconductor domain buried in a rod-shaped shell of a second semiconductor material ${ }^{13,44-46}$ and tetrapods in which either the central core region is made of a semiconductor material different from that of the arms ${ }^{45,47-49}$ or epitaxial growth of a second semiconductor material occurs selectively at the tips of the starting tetrapods. ${ }^{50,51}$ Our group recently synthesized tetrapod-shaped type-II nanocrystals of various combinations of II-VI semiconductors ${ }^{45}$ and exploited CdSe (core)/CdTe (arms) tetrapods in hybrid organic-nanocrystal solar cells. ${ }^{52,53,73}$

This report provides a study of the optical properties of CdSe (core)/CdTe (arms) tetrapods of various sizes, with the aim of determining the nature of the observed optical transitions and the main nonradiative recombination processes. Moreover, we evaluated the actual barrier height for electrons by taking into account quantum confinement effects. The knowledge of the actual barrier allowed us to analyze with higher accuracy the temperature dependence of the PL intensity, by which we could identify the thermally induced processes that reduce the emission efficiency at high temperature. The experimental results were rationalized by means of theoretical calculations, based on the envelope-function approximation (EFA), ${ }^{67}$ by which we determined the electron-hole wave function localization in the lowest confined states of the tetrapods, the relative energy separations, and the oscillator strength of both type-I and type-II optical transitions. We corroborated these results by time-resolved measurements carried out at $10 \mathrm{~K}$ (to avoid undesired thermal effects) and found evidence of the different degrees of electron-hole wave function localization.

\section{MATERIALS AND METHODS}

The CdSe (core)/CdTe (arms) tetrapods studied here were synthesized by a seeded-growth technique, using roughly 


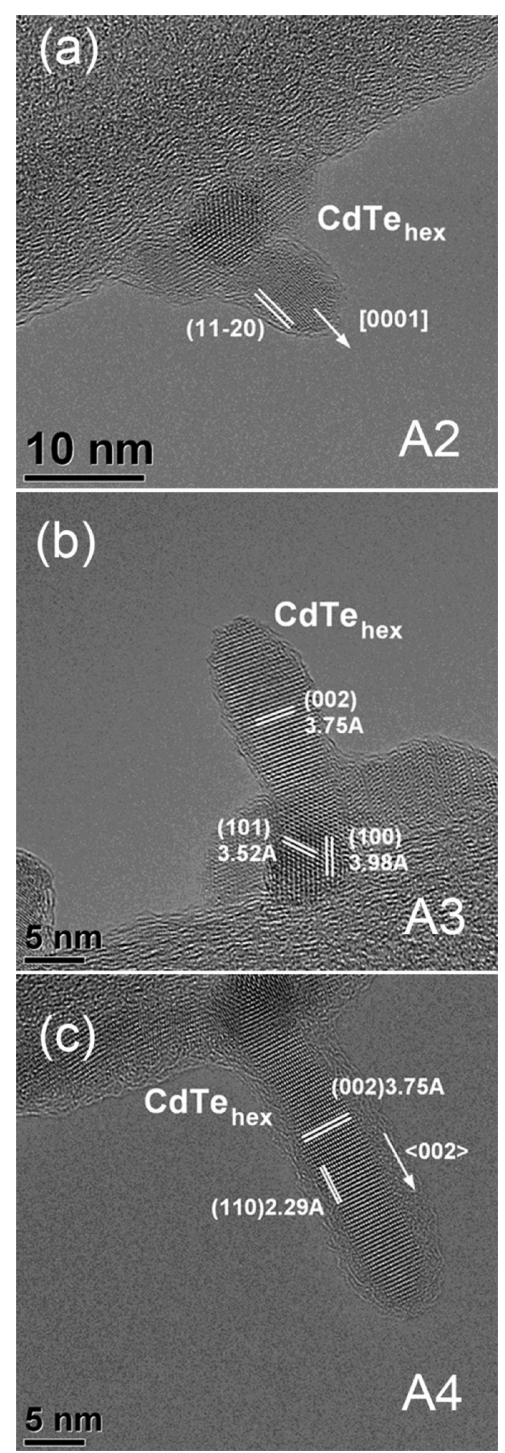

Figure 2. HRTEM images of samples (a) A2, (b) A3, and (c) A4, tilted such that all four wurtzite CdTe arms are visible. The arms grew along the $\langle 002\rangle$ direction in all three cases. The (101) and (100) planes can also be identified in image $b$, where the direction of the arm in the bottom part of the tetrapod is normal to the image plane (the arm is observed along the [001] zone axis). The images evidence the tendency for decreasing arm diameter with increasing arm length.

spherical CdSe seeds on which CdTe was subsequently grown, as described in a previous work by our group. ${ }^{45}$ Four tetrapod samples, with different core sizes and arm lengths, were synthesized and studied. The features of the various samples (core and arm dimensions, as well as the main physical quantities extracted from the analysis) are listed in Table 1. For the morphological analysis, low-magnification transmission electron microscopy (TEM) images were recorded on a JEOL JEM 1011 microscope operating at $100 \mathrm{kV}$. A detailed structural characterization [through spherical-aberration-corrected high-resolution transmission electron microscopy (HRTEM)] analysis was carried out on a JEOL 2200 FS instrument, equipped with a fieldemission electron source and operating at $200 \mathrm{kV}$. The absorption spectra of solutions containing the starting CdSe seeds and the $\mathrm{CdSe} / \mathrm{CdTe}$ tetrapods were recorded with a Cary 5 Varian
UV-vis-NIR spectrophotometer. To perform PL measurements at different temperatures in the range of $10-300 \mathrm{~K}$ and at different excitation powers (from $25 \mu \mathrm{W}$ to $34 \mathrm{~mW}$ ), a few drops of toluene solutions containing tetrapods (in the micromolar concentration range) were deposited onto a $\mathrm{SiO}_{2}$ substrate. The solvent was allowed to evaporate, and the substrate was placed in a closed-cycle $\mathrm{He}$ cryostat under vacuum conditions. An argon laser $(\lambda=514 \mathrm{~nm})$ was used to excite both the CdSe core region and the CdTe arms of the tetrapods, whereas a $\mathrm{He}-\mathrm{Ne}$ laser $(\lambda=633 \mathrm{~nm})$ was used to excite exclusively the CdTe arms. The time-correlated single-photoncounting (TCSPC) technique was used to record time-resolved $\mathrm{PL}$ in the spectral range of $0.95-1.30 \mathrm{eV}$ using a cooled NIR photomultiplier tube. The samples were excited by the $1.94 \mathrm{eV}$ $(639 \mathrm{~nm})$ line delivered by a solid-state pulsed laser, having a pulse duration of $100 \mathrm{ps}$ and a variable repetition rate in the range from $1 \mathrm{MHz}$ to $100 \mathrm{kHz}$. Time-resolved PL measurements on the type-I emission were performed at $10 \mathrm{~K}$ by exciting the tetrapods with the second harmonic $(405 \mathrm{~nm})$ of a Ti:sapphire laser having 80 -fs pulses at an $80-\mathrm{MHz}$ repetition rate. The signal was detected with a streak camera with temporal resolution of about 12 ps.

\section{MORPHOLOGICAL CHARACTERIZATION}

Transmission electron microscopy (TEM) images of the four samples are displayed in Figure 1. At first glance, the most apparent difference consists of the slimming of the arms with increasing length. Sample A1, for instance (Figure 1a), had the shortest arms $(6 \mathrm{~nm})$, and the shape of each arm resembled a pyramid rather than a prism, such that the overall shape of the nanocrystals was more like a "stretched" tetrahedron than a tetrapod. Samples A2-A4, on the other hand, had progressively longer arms, with constant diameters along the arms (Figure $1 \mathrm{~b}-\mathrm{d}$ ) and presented overall a more uniform tetrapod shape.

As a general trend, tetrapod samples with longer arms were characterized by smaller arm diameters (average diameters, for example, were 6-7 $\mathrm{nm}$ for sample $\mathrm{A} 3$ and 5-6 $\mathrm{nm}$ for sample A4), as also confirmed by HRTEM (see Figure 2).

Because the diameter is the main dimensional parameter influencing the energy gap of the tetrapods, tetrapod samples with shorter arms (hence, larger arm diameters) were actually characterized by smaller band gaps (see Figure 6c, below). The tetrapod arms were formed by hexagonal CdTe grown along its $\langle 002\rangle$ crystalline direction on top of the CdSe seeds, as shown by HRTEM (Figure $2 \mathrm{a}-\mathrm{c}$ ). In some favorable cases, it was possible to observe the normal projection ([001] zone axis) of the CdTe arms grown along the $\langle 002\rangle$ direction, as shown in Figure $2 \mathrm{~b}$. The arm structure in the samples was regular, and no stacking faults were observed. Even though the diameter of the CdSe seeds used in the synthesis of these samples was $3.7 \mathrm{~nm}$, the actual diameter of the central region of the tetrapods was comparable to that of the arms $(5-7 \mathrm{~nm})$. This implies either that a uniform, spherical $\mathrm{CdTe}$ shell was grown on top of the CdSe seed, after which four $\mathrm{CdTe}$ branched evolved or, more likely, that $\mathrm{CdTe}$ arms were first formed directly on top of the CdSe seed, and their subsequent increase in diameter buried the seed. It is difficult to identify which of the two mechanisms was operative, because we were not able to fully discern the core region from the arms by HRTEM. 

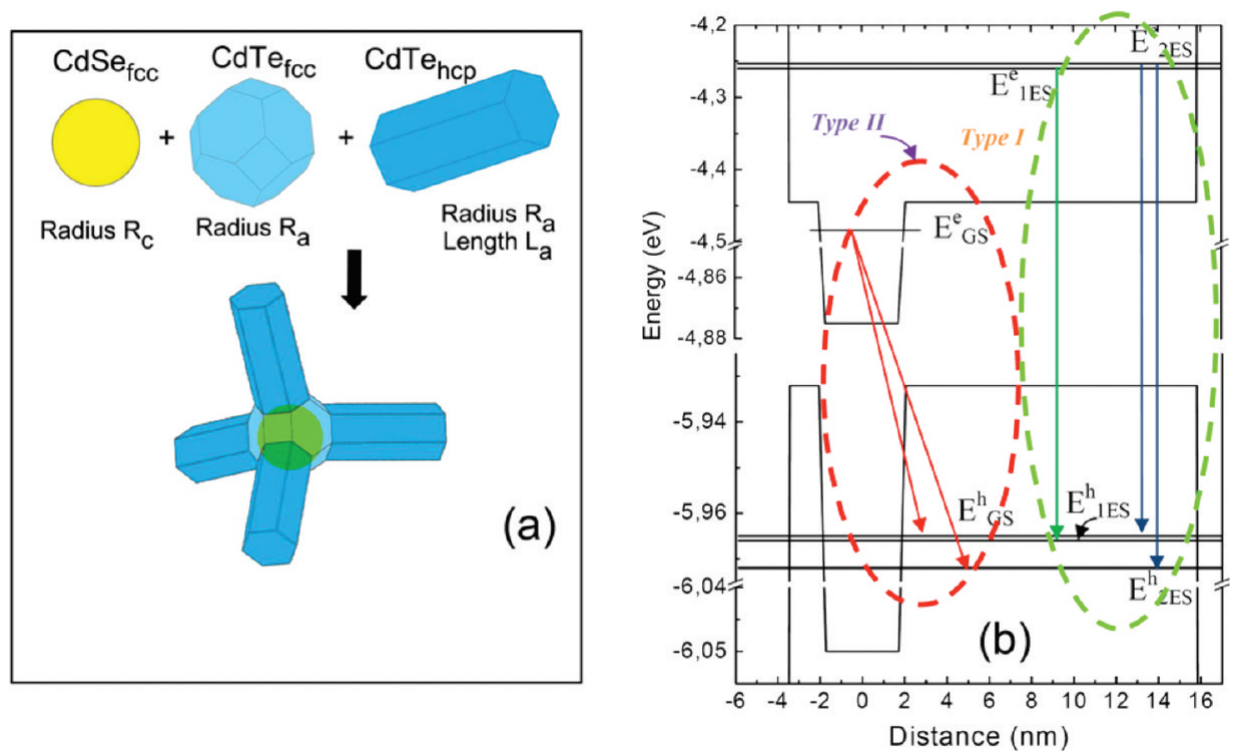

Figure 3. (a) Sketch of the tetrapod model used for the calculations. The geometrical parameters used for the calculations were derived from the HRTEM analysis and are reported here: for sample A2, $R_{\mathrm{c}}=1.8 \mathrm{~nm}, R_{\mathrm{a}}=3 \mathrm{~nm}$, and $L_{\mathrm{a}}=9 \mathrm{~nm}$; for sample A3, $R_{\mathrm{c}}=1.8 \mathrm{~nm}, R_{\mathrm{a}}=3 \mathrm{~nm}$, and $L_{\mathrm{a}}=14 \mathrm{~nm}$; and for sample A4, $R_{\mathrm{c}}=1.8 \mathrm{~nm}, R_{\mathrm{a}}=2.6 \mathrm{~nm}$, and $L_{\mathrm{a}}=23 \mathrm{~nm}$. (b) Band-edge profile along one arm direction (dashed line) and optical transitions involving the first three electron and hole confined states.
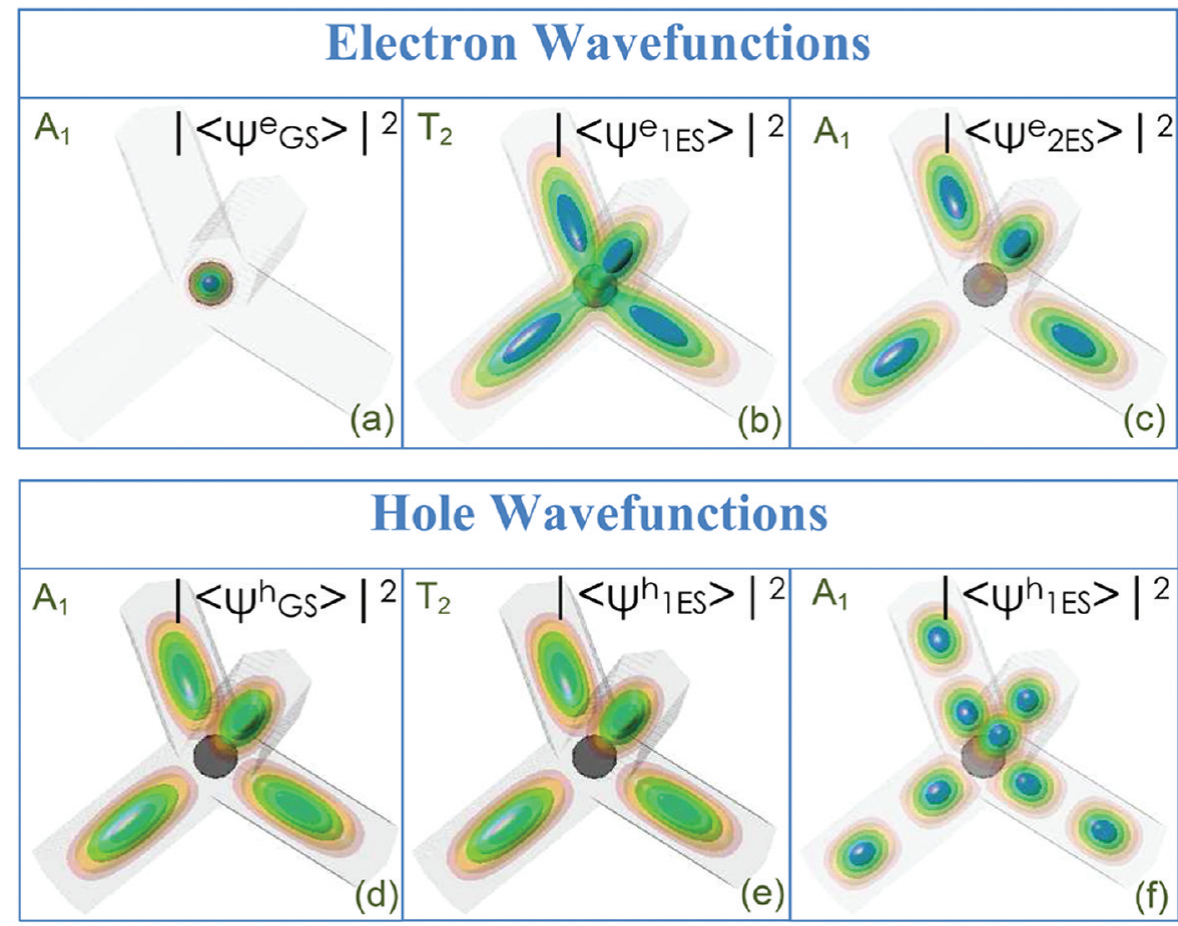

Figure 4. Three-dimensional view of the isosurfaces of $\psi^{2}$ of the first three $(\mathrm{a}-\mathrm{c})$ electron and $(\mathrm{d}-\mathrm{f})$ hole quantum states calculated for a CdSe $($ core $) /$ $\mathrm{CdTe}(\mathrm{arms})$ tetrapod with geometrical parameters equal to those of sample A3.

\section{THEORETICAL RESULTS}

Theoretical calculations based on the envelope-function approximation were performed using the in-house developed DAVMASS program ${ }^{13,58,68-71}$ to determine the electronic structure of the CdSe/CdTe tetrapods. By using in part the information derived from HRTEM measurements, we modeled the tetrapod shape by considering a CdSe sphere (of radius $R_{c}$ ) as the core inside a CdTe truncated octahedron (of dimension $R_{\mathrm{a}}$ ) from which four wurtzite (WZ) hexagonal CdTe prisms protruded as arms (of diameter $R_{\mathrm{a}}$ and length $L_{\mathrm{a}}$ ) (see Figure 3a). When considering the bulk band gap of the two different compounds at room temperature $[1.695 \mathrm{eV}$ for zincblende (ZB) CdSe and $1.487 \mathrm{eV}$ for WZ CdTe $\left.{ }^{54}\right]$, the band-edge profile along one arm direction results in a type-II system (see Figure 3b). 

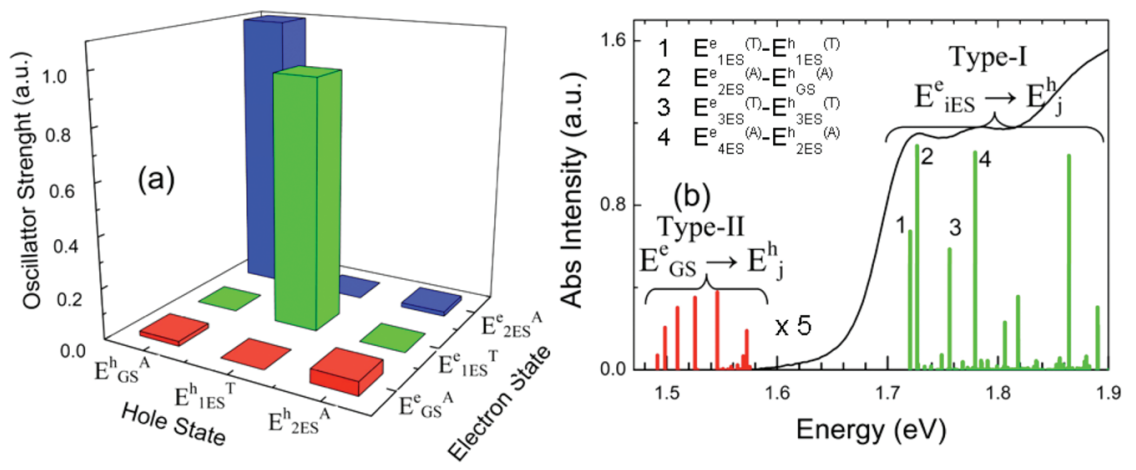

Figure 5. (a) Oscillator strengths calculated for a CdSe (core)/CdTe (arms) tetrapod with geometrical parameters equal to those of sample A3, obtained using 20 and 40 electron and hole quantized states and relative histograms. (b) Computed single-particle absorption spectrum as red (type-II transitions, magnified 5-fold) and green (type-I transitions) vertical bars for sample A3 and comparison with the experimental data (solid black line).

In the calculation, we assumed that the electrons are confined by a potential barrier whose height is equal to the electron affinity of $\mathrm{CdTe}^{13}$ whereas the holes are confined by a potential barrier of $4 \mathrm{eV}$. The variable-effective-mass Hamiltonian ${ }^{72}$ was solved on a three-dimensional cartesian grid. The employed effective masses were $m_{\mathrm{e}}=0.13$ and $m_{\mathrm{h}}=0.45$ for CdSe and $m_{\mathrm{e}}=0.11$ and $m_{\mathrm{h}}=$ 0.72 for CdTe, where subscripts e and h denote electrons and holes, respectively.

The first three confined states in the conduction and valence bands for sample A3 are shown in Figure 4. As in the case of allCdTe tetrapods, ${ }^{23}$ we found that, in the ground-state (GS) exciton, the electron density (Figure 4a) is mostly localized in the CdSe core region of the tetrapods, whereas the electron wave function spreads into the arms for the first excited state (1ES, Figure 4b), until it becomes completely delocalized into the arms for the second excited state (2ES, Figure 4c). On the other hand, the holes of the first three confined states are completely delocalized in the CdTe arms (Figure $4 \mathrm{~d}-\mathrm{f}$ ).

Moreover, we found that the electron and hole wave functions of the ground and second excited state have $A_{1}$ symmetry (Figure $4 \mathrm{a}, \mathrm{c}, \mathrm{d}, \mathrm{f}$ ), whereas the electron and hole wave functions of the first excited state have $\mathrm{T}_{2}$ symmetry (Figure $4 \mathrm{~b}, \mathrm{e}$ ).

We calculated the oscillator strengths (OSs) between the first three confined states (Figure 5a). Clearly, for transitions between states of different symmetry (such as $E_{1 \mathrm{ES}}^{\mathrm{h}} \rightarrow E_{\text {GS }}^{\mathrm{e}}$ or $E^{\mathrm{h}}{ }_{\mathrm{GS}}^{\mathrm{A}} \rightarrow$ $E_{1 E S}^{\mathrm{e}}{ }^{\mathrm{T}}$ ), they are zero. Moreover, one can also readily observe that the transitions from the electron ground state to the hole quantized states are characterized by an OS that is much smaller than those of the other transitions between the electron excited states and hole states. This is because the former are associated with type-II transitions (shown in Figure 5a as red histograms) between the different hole confined states (for which the carriers are mainly delocalized into the tetrapod arms; see Figure $4 d-f$ ) and the electron ground state localized in the core (Figure 4a). Instead, the other transitions are of type-I (green and blue histograms in Figure 5a) and involve electron and hole confined states of carriers delocalized in the CdTe arms.

In Figure $5 b$, we report the single-particle absorption spectrum, computed for sample A3 including transitions between the 40 lowest hole states and the 20 lowest electron states, superimposed on the experimental absorption spectrum. The main absorption onset with two superimposed peaks originates from the convolution of the first four type-I transitions having the largest oscillator strengths (and identified as green bars). In particular, those labeled 1 and 2 (having a spacing of about $6 \mathrm{meV}$ ) are transitions between the first two electron excited states and the two lowest, degenerate, hole states. The one labeled as 3 is determined by the transition between the third excited electron state and the third hole state, and it lies at about $36 \mathrm{meV}$ from transition 2. Finally, the fourth electron excited state and the second hole excited state produce the transition labeled 4 at about $59 \mathrm{meV}$ from the transitions 1 and 2 . The red bars at lower energy in Figure 5b, characterized by weak oscillator strengths, are associated with type-II transitions (these are shown in Figure 5a for the first three confined states as red histograms). The large difference in the OSs between the type-II and type-I transitions together with the different symmetries of the confined states should clearly affect the photoluminescence (PL) spectra of these $\mathrm{CdSe} / \mathrm{CdTe}$ tetrapods. In particular, even though the lowest-energy transition should be an infrared type-II transition of the type $E_{\text {GS }}^{\mathrm{e}} \rightarrow E_{\text {GS }}^{\mathrm{h}}$, a finite probability for the higher-energy transition $E_{1 \mathrm{ES}}^{\mathrm{e}} \rightarrow E_{1 \mathrm{ES}}^{\mathrm{h}}$, as a type-I transition with electrons and holes delocalized along the arms, should be taken into account, because the probability of an intraband transition leading the carriers from the first excited state, $E_{1 \mathrm{ES}}$, with $\mathrm{T}_{2}$ symmetry to the ground state, $E_{\mathrm{GS}}$, with $\mathrm{A}_{1}$ symmetry, is very small or even zero.

\section{OPTICAL CHARACTERIZATION AND DISCUSSION}

The tetrapod absorption spectra (Figure 6a) exhibited a clear red shift with respect to that of the CdSe seeds (Figure 6b), because of the absorption of the CdTe arms, whose volume is much greater than that of the CdSe seeds (in our tetrapods, $V_{\text {seed }} / V_{\text {arms }}$ ranges from 0.2 to 0.05 for different dimensions). Unlike samples A3 and A4, samples A1 and A2 showed less structured and broader spectra, typical of nanostructures in which the type-II nature becomes observable in absorption. Indeed, in these samples (especially sample A1), the volumes of the two components were comparable. The appearance of a long red tail is a signature of the dominance of type-II absorption. $^{38,50,56}$ The corresponding PL spectra (Figure 6c) exhibit a broad peak located in the near-IR region (labeled as P2 peak), about $400 \mathrm{meV}$ below the absorption band edge of the tetrapods. The PL line shape has an asymmetric character, and the full width at half-maximum (fwhm) of this signal is generally on the order of $150 \mathrm{meV}$, well above the usual broadening observed in type-I colloidal nanocrystals (about 70-80 $\mathrm{meV}^{66}$ ), exhibiting an almost monotonic behavior (A1, $138 \mathrm{meV}$; A2, 165 $\mathrm{meV}$; A3, $152 \mathrm{meV}$; A4, $170 \mathrm{meV}$ ). These values closely follow those recently reported on $\mathrm{CdTe} / \mathrm{CdSe}$ type-II nanocrystal 

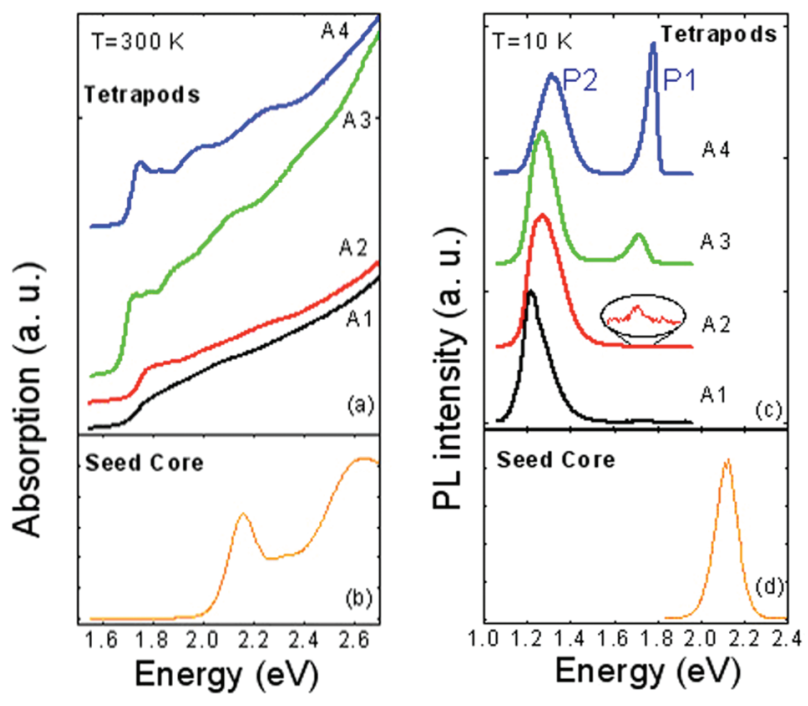

Figure 6. Absorption spectra of the (a) four samples of tetrapods and (b) CdSe starting seeds (orange line), as recorded at room temperature. PL spectra of the (c) four tetrapods and (d) CdSe starting seeds measured at $10 \mathrm{~K}$. The presence of type-I (P1) and type-II (P2) peaks in longer-arms tetrapods is noticeable, along with the increasing contribution of the visible emission and the blue shift of both $\mathrm{P} 1$ and $\mathrm{P} 2$ with increasing arm length. Peak P1 of sample A2 has been magnified for clarity.

systems by de Mello Donegá ${ }^{38}$ for which the broadening was found to increase with increasing volume of the two individual components of the heterostructure. The asymmetry of the PL is typical for type-II heterostructures, and it is commonly ascribed to the inhomogeneity of the samples and to the unavoidable multiplicity of the type-II system, as found by our calculations as well (see Figure 5b). In addition, by fitting the type-II PL with a number of Gaussian peaks (not shown here), we found that each type-II peak can be considered as a convolution of at least three Gaussian peaks. We therefore consider both of these effects as being responsible for the asymmetric line shape observed.

Only tetrapod samples with arms longer than $6 \mathrm{~nm}$ exhibited an additional PL peak (labeled as P1), which fell in a region close to the lowest excitonic absorption peak (indeed, sample A2 shows a very low signal, as magnified in Figure 6c). As for type-II emission, the $\mathrm{P} 1$ peak shows a certain asymmetry constituted by a red tail (Figure 6c). In this case, it can be ascribed to a very common process afflicting the PL of colloidal nanocrystals having a single size distribution, which is the long-range resonant transfer (LRRT) of the charge excitation from nanocrystals having large energy gaps to nanocrystals with a smaller gaps belonging to the same sample. ${ }^{66,76}$

The spectral positions of P1 and P2 are strongly correlated with the size of the CdTe arms of the tetrapods. In accordance with the HRTEM observations that longer-arm samples also have smaller arm diameters, the emission energy blue shifts with increasing arm length of the tetrapods (see Table 1). Regarding the origin of the two emission peaks, the calculations reported in the previous section, together with previous experimental studies on $\mathrm{CdSe} / \mathrm{CdTe}$ nanorod heterostructures, ${ }^{58-60}$ suggest that peak P1 arises from a type-I recombination occurring in the CdTe section, whereas peak P2 arises from a type-II recombination of electrons localized in the CdSe section and holes delocalized in the CdTe section. We confirmed this attribution

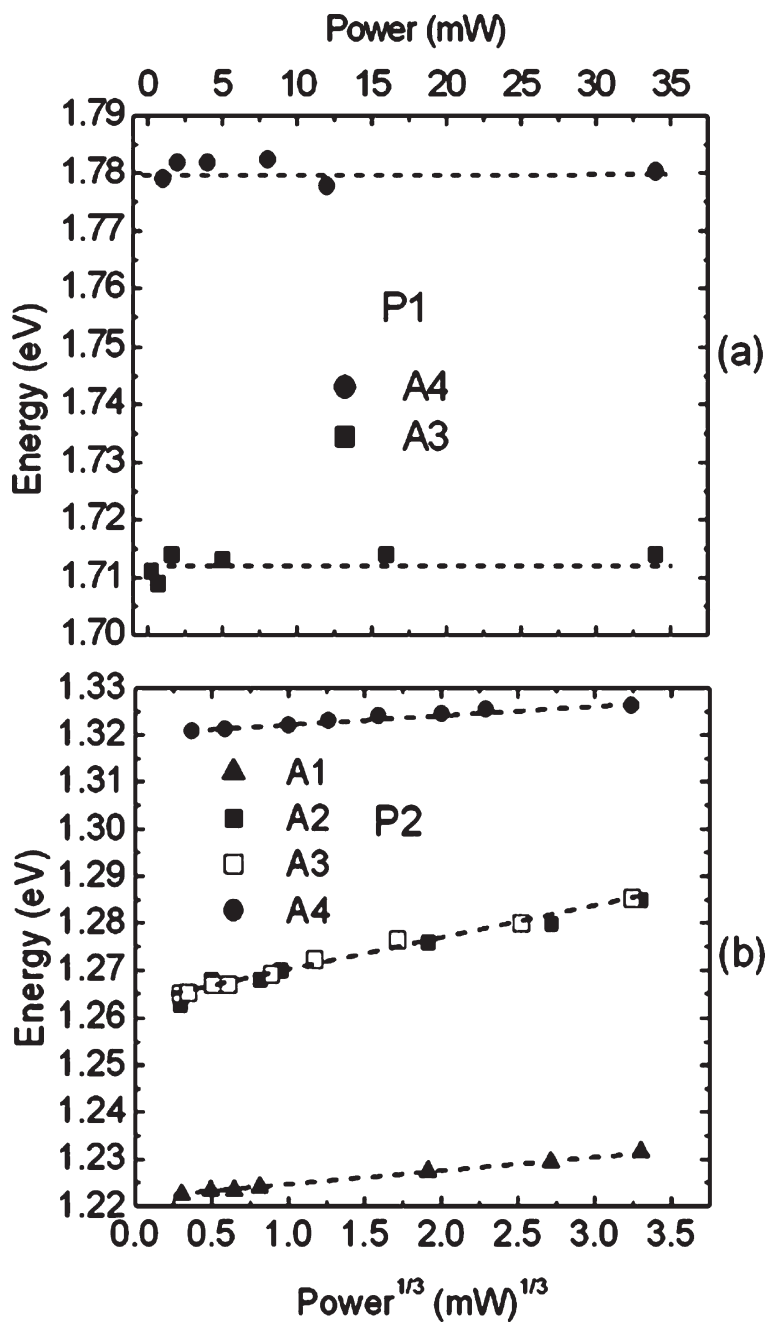

Figure 7. (a) Power dependence of the type-I emission energy of two of the four samples studied, upon excitation of the tetrapods in the power range from $25 \mu \mathrm{W}$ to $34 \mathrm{~mW}$. (b) Type-II PL energy versus the cubic root of the excitation power of the four samples. The lines are a guide for the eyes.

by performing PL experiments as a function of the excitation power. In Figure 7, we plot the energy positions of peaks P1 and $\mathrm{P} 2$ as a function of the excitation power for the samples studied. In particular, Figure $7 \mathrm{~b}$ reports the evolution of the P2 energy versus the cubic root of the applied excitation power (ranging from $25 \mu \mathrm{W}$ to $34 \mathrm{~mW}$ ). The signals originating from $\mathrm{A} 2$ and $\mathrm{A} 3$ are almost completely overlapped because of the proximity of their type-II emission energies. A clear blue shift of the emission energy with increasing laser power was observed (total shifts of 9, 22, 20.4, and $5.4 \mathrm{meV}$ for samples A1-A4, respectively). Such a linear dependence of the shift on the cube root of the power represents a clear fingerprint of a type-II transition. ${ }^{61}$ This is due to a screening of the internal electric field generated by the charge separation under intense photogeneration in the neighborhood of the interface, because of the storage of electrons and holes near the core/arm interface. ${ }^{61,62}$ This field induces a band bending near the contact surface between the two materials and a consequent blue shift of the original type-II transition, because the transition originates mainly from recombination of carriers located at the interfacial region. ${ }^{62}$ In contrast, no detectable shift 
and/or a linear dependence on the cubic root of the laser power was observed for the P1 peak energy (Figure 7a), which had the typical behavior of a type-I transition. In addition, it is interesting to note that the data of Figure 7 are consistent with the size dependence of the energy gap of the arms, whose diameter shrinks with increasing length. In the $\mathrm{CdSe} / \mathrm{CdTe}$ type-II nanorods synthesized by other groups, the presence of the visible CdTe peak was explained by assuming two main processes: ${ }^{58}$ (i) resonant energy transfer (RET) from higher-energy-gap CdSe to lower-gap CdTe and (ii) ultrafast core-arm hole transfer. Considering the energy transfer from CdSe to CdTe to be responsible for the CdTe emission, we note that it should be measured in both long-arm (as actually noticed) and short-arm tetrapods. This is in contrast to our experimental evidence, according to which the type-I transition was suppressed or considerably decreased in samples A1 and A2. On the other hand, ultrafast hole transfer would require the rate of hole transfer to be several orders of magnitude higher than the rate of electron transfer. PL experiments (not shown here) performed by exciting the tetrapods exclusively in the CdTe arms with a $\mathrm{He}-\mathrm{Ne}$ laser at $1.958 \mathrm{eV}$ (i.e., at an energy below the CdSe core energy gap and thus excluding both core-arm RET and hole

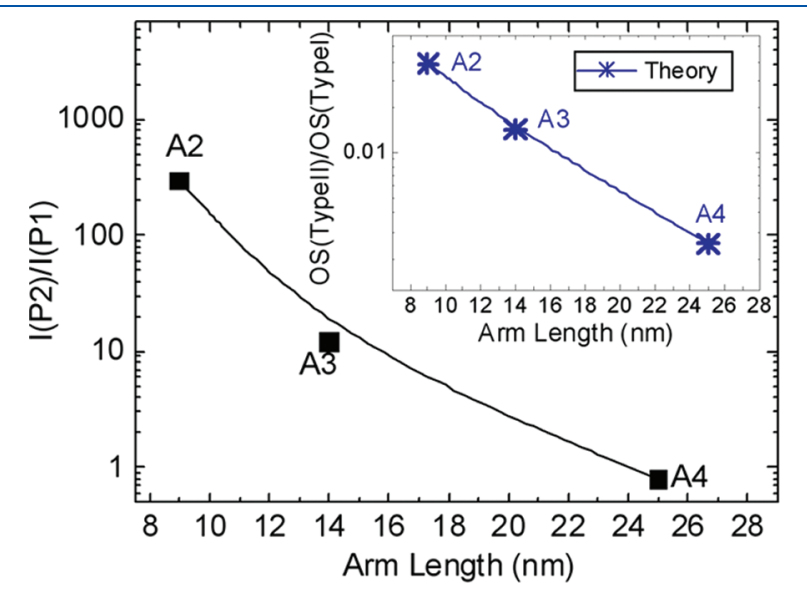

Figure 8. Ratios of the PL integrated areas of the two emission peaks plotted for three of the samples studied, as a function of the arm length (on a semilogarithmic scale). The lines are a guide for the eyes. Inset: Ratio of the oscillator strengths of the type-II transition to that of the type-I transition (according to calculations) for samples A2-A4 as a function of the arm length. transfer from CdSe to the CdTe valence band) showed that the visible PL peak was still preserved. This suggests that, even though RET and ultrafast hole transfer might play some role in our tetrapods, they cannot fully explain our experimental data. Instead, the presence of the type-I transition can be better justified by the different symmetries featuring the two observed optical transitions, as discussed in the Theoretical Results section.

From PL spectra obtained for samples with the same nanocrystal concentration and under equal excitation conditions, we also observed a variation in the relative integrated area of the two PL emission peaks as a function of the tetrapod size. By plotting the ratio of the integrated area of the $\mathrm{P} 2$ and $\mathrm{P} 1$ emission peaks (associated with a type-II transition and a type-I transition, respectively) for all of the studied samples, we observed a decrease in the relative intensity of P2 with respect to P1 as a function of the arm length (Figure 8). Theoretical calculations performed using geometrical parameters corresponding to the values for samples $\mathrm{A} 2-\mathrm{A} 4$ showed the same qualitative trend in plots of the ratio of the oscillator strength of the type-II transition to that of the type-I transition as a function of the arm length (inset of Figure 8). This can be explained by the fact that, for a constant core size, an increase in the arm length results in a decrease in the electron-hole overlap in the ground state (i.e., a reduction in the OS of the type-II transition), whereas the electron-hole overlap of the first excited state remains quite constant (implying a negligible variation of the OS of the type-I transition). To obtain experimental support for these theoretical results, we performed time-resolved PL measurements at $10 \mathrm{~K}$ on all four tetrapod samples. The time traces were fitted to the equation

$$
I(t)=\sum_{i=1}^{n} A_{i} \mathrm{e}^{-\left(t-t_{0}\right) / \tau_{i}}
$$

This sum accounts for the multiexponential character of the decays, where $\tau_{i}$ is the lifetime of the $i$ th decay process and $A_{i}$ represents its relative contribution to the overall decay. The typeI emission (recorded in a time window of 2 ns, not reported here) decays in a nonexponential way (triexponential) with an average lifetime of some hundreds of picoseconds. Such properties, extensively studied in ref 63, are typical of all-CdTe tetrapodshaped colloidal nanocrystals having a double emission peak, in which the higher-energy emission closely follows the characteristics
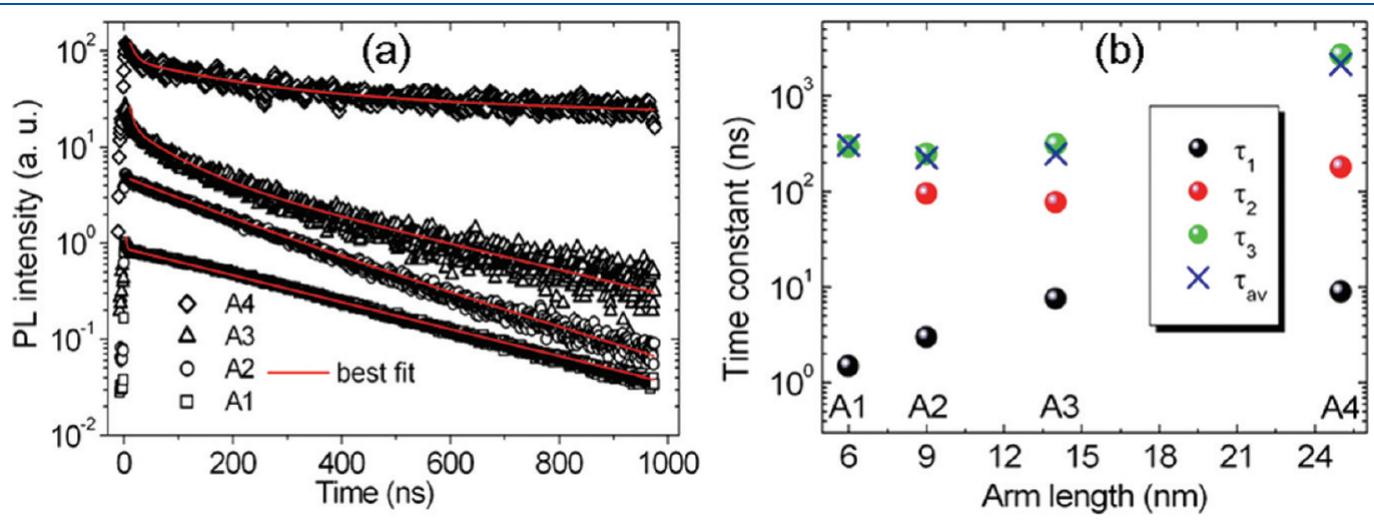

Figure 9. (a) Time-resolved PL traces of the four samples recorded at $10 \mathrm{~K}$, showing multiexponential decays (symbols), and relative best fit to eq 1 (red lines). (b) Plot of the single time constants extracted by the analysis $\left(\tau_{1}-\tau_{3}\right)$ and the average lifetime $\left(\tau_{\mathrm{av}}\right)$ as a function of the arm length for the four samples studied. 

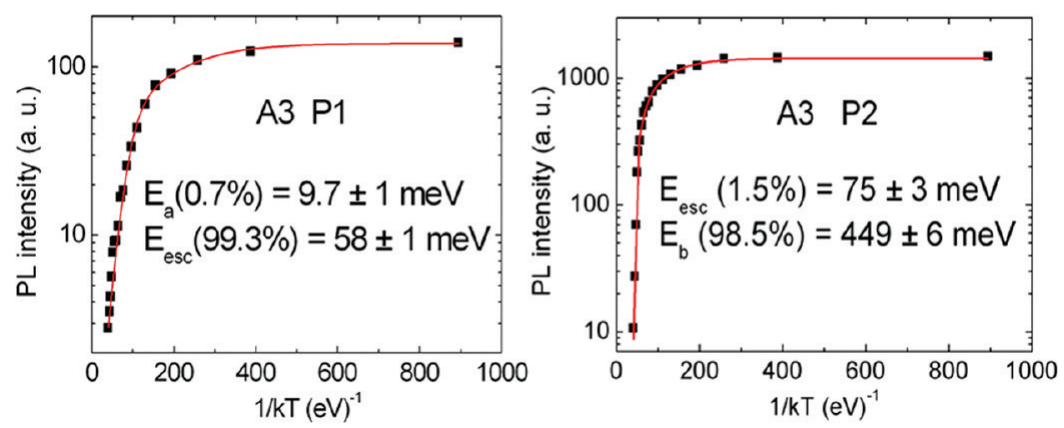

Figure 10. Plots of the integrated PL intensities versus $1 / k_{\mathrm{B}} T$ (symbols) of both P1 and P2 for sample A3 and relative best fit curves (red lines) to eq 3 . The percentage values in parentheses indicate the relative contributions of the single processes considered.

of the type-I peak emission of our tetrapods. Here, we focus our attention on the type-II transition with the aim of verifying that tetrapods having different arm lengths had lifetimes that varied according to the peculiar electron-hole localization.

We studied such decay times in a temporal window of $1 \mu \mathrm{s}$, as we expected lifetimes longer than those of type-I PL, because of the indirect nature of the transition. In Figure 9, we report the temporal decays for the four samples (Figure 9a) and the extracted time constant values for each sample (Figure 9b). For completeness, in the same figure, we report the average lifetimes as well, extracted using the expression

$$
\tau_{\mathrm{av}}=\frac{\sum_{i=1}^{n} A_{i} \tau_{i}^{2}}{\sum_{i=1}^{n} A_{i} \tau_{i}}
$$

We note that almost all of the samples exhibit triexponential decay, except for sample A1, which presents a biexponential behavior. Both the single and average lifetimes follow a clear increasing trend with increasing arm length, apart from a slight deviation for sample A1, which decays biexponentially. This behavior is consistent with the previous theoretical results, according to which increasing the arm length of the tetrapod results in a decrease of the OS of the type-II transition, thus leading to an increase in the decay time. The nonexponential decay of the type-II transition can be tentatively ascribed to radiative recombination from different intrinsic states, as reported by de Mello Donegá ${ }^{38}$ for similar materials. Based on our calculations and evidenced by steady-state PL measurements, we expect that the type-II transition is a combination of a manifold of transitions falling in the same spectral region (see Figure 5b) and involving states characterized by different degrees of electronhole localization. This is apparent, for instance, when considering the first three quantized states depicted in Figure 4: two of these states $\left(E_{\mathrm{GS}}^{\mathrm{e}} \rightarrow E^{\mathrm{h}}{ }_{\mathrm{GS}}\right.$ and $\left.E_{\mathrm{GS}}^{\mathrm{e}} \rightarrow E^{\mathrm{h}}{ }_{2 \mathrm{ES}}\right)$ give rise to two type-II transitions corresponding to two different degrees of localization for the electron and hole wave functions. At the same time, this implies that the lifetimes of these states should be different (for example, transition $E_{\text {GS }}^{\mathrm{e}} \rightarrow E^{\mathrm{h}}{ }_{2 \mathrm{ES}}$ is expected to be faster than transition $E_{\mathrm{GS}}^{\mathrm{e}} \rightarrow E_{\mathrm{GS}}^{\mathrm{h}}$ because of its larger OS, as shown in Figure 5a). The nonexponential character of the temporal decays of all of the samples can therefore be associated to the detection of a signal coming from a number of different states responsible for the type-II PL. As extracted by the PL analysis, the type-II peaks of all of the samples studied can be considered as convolutions of at least three emitting states, although the real number could be greater, as depicted in Figure 5. For these reasons, the three exponential decays can be associated with the lifetimes of the single emitting states featuring each sample. Regarding sample A1, the absence of one component could be indicative of some minor structural and/or geometrical differences between that sample and all of the others, as can be guessed by the TEM analysis (Figures 1 and 2), which would then be responsible for differences in the electronic structure. These hypotheses, however, need to be confirmed by more detailed calculations and experiments (which are currently in progress and which will be the focus of a future work).

Another important issue is the understanding of the thermally activated processes, acting as nonradiative channels that quench the PL at high temperature. Toward this end, we analyzed the temperature dependence of the PL intensity in all of our samples.

In Figure 10, we report the results of this analysis for a representative sample, namely, sample A3. As generally observed in colloidal nanocrystals, the PL intensity exhibits a considerable decrease at temperatures higher than $100-150 \mathrm{~K}$. The plot of the intensity as a function of $1 / k_{\mathrm{B}} T$ (where $k_{\mathrm{B}}$ is the Boltzmann constant and $T$ is the temperature) on a semilogarithmic scale allows for the determination of the activation energy of the thermal processes triggered at high temperature, by fitting the experimental data with an Arrhenius law, using the expression

$$
I(T)=\frac{I_{0}}{1+\sum_{i=1}^{n} a_{i} \mathrm{e}^{\left(-E_{i} / k_{\mathrm{B}} T\right)}}
$$

Here, $n$ is the number of thermal processes, $E_{i}$ represents the activation energy of the $i$ th process, and $a_{i}$ is a fitting parameter accounting to the relative weight of each exponential term. For each sample, we separately considered the PL arising from a typeI recombination in the $\mathrm{CdTe}$ arms (P1 transition) and the signal due to the indirect type-II recombination (P2). The activation energies of the different samples are summarized in Table 1. For the sake of clarity, we limit this discussion to the sample of Figure 10, although all of our conclusions apply to the other tetrapod samples as well. Concerning the type-I emission (Figure 10a, peak P1) originating from the CdTe arms, this is characterized by two thermal processes, whose activation energies are, for sample A3, equal to 9.7 and $58 \mathrm{meV}$. Even though only two samples included in Table 1 show the smallest activation energy (labeled as $E_{\mathrm{a}}$ ), this quantity characterized a larger number of samples (not shown here) with a minor contribution and was randomly distributed in the $7.5-14 \mathrm{meV}$ range with 
apparently no correlation with the morphology of the samples (size and/or shape). Typical exciton binding energies in CdTe, as reported in the literature, range from 17.5 to $25 \mathrm{meV}$. Therefore, the process characterized by the lower activation energy in our tetrapods is due not to exciton ionization but rather to carrier delocalization from surface and/or core-arm interface defect states. ${ }^{66}$ The process activated at higher temperature (with activation energy $E_{\text {esc }}$ ) exhibits a diameter-dependent behavior consisting of increasing activation energy with decreasing arm diameter. This suggests that the process is associated with the degree of quantization of the system and then with the energy spacing among the states. In particular, we note that $E_{\text {esc }}$ follows strictly the same trend of $\Delta E_{1,2}$, which is the energy separation between the first two well-resolved absorption peaks, as evidenced in Table 1. To assess the clear origin of this thermal process, we compared the experimental and theoretical absorption spectra, as depicted in Figure 5b. Considering the theoretical absorption shown in Figure 5, one can regard the pairs of transitions 1, 2 and 3, 4 (hereafter referred to as T1 and T2, respectively), as the highest contributors to the absorption at lower energy. Therefore, by taking into account their relative contributions, we can extract an average energy separation between the two couples so that the value of $\Delta E_{1,2}$ reported in Table 1 can be reasonably attributed to that value (about $49 \mathrm{meV}$ in sample A3). The size dependence and the similarity between $E_{\text {esc }}$ and $\Delta E_{1,2}$ suggest that the quenching process is related to carriers jumping from states belonging to the pair $\mathrm{T} 1$ to states belonging to the pair T2 (i.e., thermal escape). ${ }^{65,66}$ Indeed, this process (characterized by carrier-phonon coupling) is strictly related to the degree of quantization, because it induces the jump of the carriers between the different confined states through absorption of a certain number of optical phonons such as to cover the energy spacing, and it is known to be the main cause of temperature-induced PL quenching in colloidal nanocrystals. ${ }^{65,66}$ The P2 (type-II) transition presents two activation energies (75 and $449 \mathrm{meV}$ ), although the first one has a very low contribution (about $1 \%$ in all of the samples studied). Whereas the first energy can be reasonably ascribed to thermal escape, as in a standard type-I transition (strongly attenuated because of the electron localization into the core), the second energy $\left(E_{\mathrm{b}}\right.$ in Table 1$)$ provides a signature of electron scavenging across the conduction band barrier, $\Delta E_{\mathrm{C}}$, from the CdSe seed to one of the CdTe arms, which can be defined as the energy spacing between type-I and type-II transitions $\left(\Delta E_{\mathrm{I}-\mathrm{II}}\right.$ in Table 1$)$. The likely occurrence of this process is supported both by the results of our computations, according to which the first conduction band excited state in CdSe seeds should lie above several hundred millielectronvolts, and by the agreement between the analytical activation energy $E_{\mathrm{b}}$ and $\Delta E_{\mathrm{I}-\mathrm{II}}$.

\section{- CONCLUSIONS}

In summary, we have studied the optical properties of CdSe (core)/CdTe (arm) tetrapods synthesized by a seeded-growth approach. We found that, because of band alignment, the nanostructures exhibit a type-II transition falling in the infrared region and a type-I visible transition arising from direct recombination occurring exclusively in the CdTe arms. Short-arm tetrapods (shorter than $9 \mathrm{~nm}$ ) show the indirect type-II transition only, whereas for longer arms, the direct transition survives. Aided by theoretical considerations, we assigned the origin of the visible emission and explained its presence in terms of the different symmetries of the two involved transitions (which cause a reduction of the intraband carrier relaxation and a nonzero probability of the higher-energy type-I optical transition occurring in the CdTe arms). We probed this hypothesis by performing time-resolved PL measurements on both type-I and type-II emissions, finding good evidence for the various degrees of electron-hole wave function localization occurring in tetrapods with different arm lengths. The thermally activated nonradiative processes responsible for the reduced PL efficiency at high temperature were also studied. The type-I transition undergoes a main nonradiative process consisting of thermal escape of both electron and hole carriers. Furthermore, the CdSe/CdTe tetrapods are characterized by an additional process that is activated at low temperatures (a few millielectronvolts) and that is likely responsible for carrier delocalization along the arms. Finally, we found that the type-II transition sustains electron promotion from the core region to the arm conduction band.

\section{AUTHOR INFORMATION}

\section{Corresponding Author}

*E-mail: giovanni.morello@unisalento.it. Tel.: +39 0832295710. Fax +390832 295708 .

\section{ACKNOWLEDGMENT}

The authors gratefully thank P. Cazzato for valuable technical assistance. This work was financially supported in part by the FP7 starting ERC grant NANO-ARCH (contract no. 240111).

\section{REFERENCES}

(1) Anikeeva, P. O.; Halpert, J. E.; Bawendi, M. G.; Bulović, V. Quantum Dot Light-Emitting Devices with Electroluminescence Tunable Over the Entire Visible Spectrum. Nano Lett. 2009, 9, 2532-2536.

(2) Caruge, J. M.; Halpert, J. E.; Wood, V.; Bulović, V.; Bawendi, M. G. Colloidal Quantum-Dot Light-Emitting Diodes with Metal-Oxide Charge Transport Layers. Nat. Phot. 2008, 2, 247-250.

(3) Chan, Y.; Caruge, J. M.; Snee, P. T.; Bawendi, M. G. Multiexcitonic Two-State Lasing in a CdSe Nanocrystal Laser. Appl. Phys. Lett. 2004, 85, 2460-2462.

(4) Klimov, V. I.; Ivanov, S. A.; Nanda, J.; Achermann, M.; Bezel, I.; McGuire, J. A.; Piryatinski, A. Single-Exciton Optical Gain in Semiconductor Nanocrystals. Nature 2007, 447, 441-446.

(5) Klimov, V. I.; Mikhailovky, A. A.; Xu, S.; Malko, A.; Hollingsworth, J. A.; Leatherdale, C. A.; Eisler, H. -J.; Bawendi, M. G. Optical Gain and Stimulated Emission in Nanocrystal Quantum Dots. Science 2000, 290, 314-317.

(6) Lee, Y.-L.; Chi, C.-F.; Liau, S.-Y. CdS/CdSe Co-Sensitized $\mathrm{TiO}_{2}$ Photoelectrode for Efficient Hydrogen Generation in a Photoelectrochemical Cell. Chem. Mater. 2010, 22, 922-927.

(7) Sun, B. Q.; Marx, E.; Greenham, N. C. Photovoltaic Devices Using Blends of Branched CdSe Nanoparticles and Conjugated Polymers. Nano Lett. 2003, 3, 961-963.

(8) Deka, S.; Quarta, A.; Lupo, M. G.; Falqui, A.; Boninelli, S.; Giannini, C.; Morello, G.; De Giorgi, M.; Lanzani, G.; Spinella, C.; Cingolani, R.; Pellegrino, T.; Manna, L. CdSe/CdS/ZnS Double Shell Nanorods with High Photoluminescence Efficiency and Their Exploitation As Biolabeling Probes. J. Am. Chem. Soc. 2009, 131, 2948-2958.

(9) Michalet, X.; Pinaud, F.; Lacoste, T. D.; Dahan, M.; Bruchez, M. P.; Alivisatos, A. P.; Weiss, S. Properties of Fluorescent Semiconductor Nanocrystals and their Application to Biological Labeling. Single Mol. 2001, 4, 261-276.

(10) Li, J.; Wang, L. -W. Shape Effects on Electronic States of Nanocrystals. Nano Lett. 2003, 3, 1357-1363. 
(11) Hewa-Kasakarage, N. N.; Kirsanova, M.; Nemchinov, A.; Schmall, N.; El-Khoury, P. Z.; Tarnovsky, A. N.; Zamkov, M. Radiative Recombination of Spatially Extended Excitons in ( $\mathrm{ZnSe} / \mathrm{CdS}) / \mathrm{CdS}$ Heterostructured Nanorods. J. Am. Chem. Soc. 2009, 131, 1328-1334.

(12) Krahne, R.; Morello, G.; Figuerola, A.; George, C.; Deka, S.; Manna, L. Physical Properties of Elongated Inorganic Nanoparticles. Phys. Rep. 2011, 501, 75-221.

(13) Carbone, L.; Nobile, C.; De Giorgi, M.; Della Sala, F.; Morello, G.; Pompa, P.; Hytch, M.; Snoeck, E.; Fiore, A.; Franchini, I. R.; Nadasan, M.; Silvestre, A. F.; Chiodo, L.; Kudera, S.; Cingolani, R.; Krahne, R.; Manna, L. Synthesis and Micrometer-Scale Assembly of Colloidal CdSe/CdS Nanorods Prepared by a Seeded Growth Approach. Nano Lett. 2007, 7, 2942-2950.

(14) Kraus, R. M.; Rogach, A. L.; Talapin, D. V.; Weller, H.; Lupton, J. M.; Feldmann, J. Room-Temperature Exciton Storage in Elongated Semiconductor Nanocrystals. Phys. Rev. Lett. 2003, 98, 017401-017404.

(15) Müller, J.; Lupton, J. M.; Rogach, A. L.; Feldmann, J.; Talapin, D. V.; Weller, H. Monitoring Surface Charge Movement in Single Elongated Semiconductor Nanocrystals. Phys. Rev. Lett. 2004, 93, 167402-167405.

(16) Sanyal, A.; Bala, T.; Ahmed, S.; Singh, Ajay; Piterina, A. V.; McGloughlin, T. M.; Laffir, F. R; Ryan, K. M. Water Dispersible Semiconductor Nanorod Assemblies via a Facile Phase Transfer and Their Application As Fluorescent Biomarkers. J.Mater. Chem. 2009, 19, 8974-8981.

(17) Baker, J. L.; Widmer-Cooper, A.; Toney, M. F.; Geissler, P. L.; Alivisatos, A. P. Device-Scale Perpendicular Alignment of Colloidal Nanorods. Nano Lett. 2010, 10, 195-201.

(18) Baranov, D.; Fiore, A.; van Huis, M.; Giannini, C.; Falqui, A.; Lafont, U.; Zandbergen, H.; Zanella, M.; Cingolani, R.; Manna, L. Assembly of Colloidal Semiconductor Nanorods in Solution by Depletion Attraction. Nano Lett. 2010, 10, 743-749.

(19) Pang, Q.; Zhao, L.; Cai, Y.; Nguyen, D. P.; Regnault, N.; Wang, N.; Yang, S.; Ge, W.; Ferreira, R.; Bastard, G.; Wang, J. CdSe Nanotetrapods: Controllable Synthesis, Structure Analysis, and Electronic and Optical Properties. Chem. Mater. 2005, 17, 5263-5267.

(20) Peng, P.; Milliron, D. J.; Hughes, S. M.; Johnson, J. C.; Alivisatos, A. P.; Saykally, R. J. Femtosecond Spectroscopy of Carrier Relaxation Dynamics in Type II CdSe/CdTe Tetrapod Heteronanostructures. Nano Lett. 2005, 5, 1809-1813.

(21) Wang, L. W. Charging Effects in a CdSe Nanotetrapod. J. Phys. Chem. B 2005, 109, 23330-23335.

(22) Malkmus, S.; Kudera, S.; Manna, L.; Parak, W. J.; Braun, M. Electron-Hole Dynamics in CdTe Tetrapods. J. Phys. Chem. B 2006, 110, 17334-17338.

(23) Tarì, D.; De Giorgi, M.; Pompa, P. P.; Carbone, L.; Manna, L.; Kudera, S.; Cingolani, R. Exciton Transitions in Tetrapod-Shaped CdTe Nanocrystals Investigated by Photomodulated Transmittance Spectroscopy. Appl. Phys. Lett. 2006, 89, 094104-094106.

(24) Al Salman, A.; Tortschanoff, A.; Mohamed, M. B.; Tonti, D.; van Mourik, F.; Chergui, M. Temperature Effects on the Spectral Properties of Colloidal CdSe Nanodots, Nanorods, and Tetrapods. Appl. Phys. Lett. 2007, 90, 093104-093106.

(25) Nobile, C.; Kudera, S.; Fiore, A.; Carbone, L.; Chilla, G.; Kipp, T.; Heitmann, D.; Cingolani, R.; Manna, L.; Krahne, R. Confinement Effects on Optical Phonons in Spherical, Rod-, and Tetrapod-Shaped Nanocrystals Detected by Raman Spectroscopy. Phys. Status Solidi A 2007, 204, 483-486.

(26) Fang, L.; Park, J. Y.; Cui, Y.; Alivisatos, A.; Shcrier, J.; Lee, B.; Wang, L.-W.; Salmeron, M. Mechanical and Electrical Properties of CdTe Tetrapods Studied by Atomic Force Microscopy. J. Chem. Phys. 2007, 127, 184704-184709.

(27) Liu, H. T.; Alivisatos, A. P. Preparation of Asymmetric Nanostructures through Site Selective Modification of Tetrapods. Nano Lett. 2004, 4, 2397-2401.

(28) Mokari, T.; Rothenberg, E.; Popov, I.; Costi, R.; Banin, U. Selective Growth of Metal Tips onto Semiconductor Quantum Rods and Tetrapods. Science 2004, 304, 1787-1790.
(29) Zhou, Y.; Li, Y. C.; Zhong, H. Z.; Hou, J.; Ding, Y.; Yang, C.; Li, Y. Hybrid Nanocrystal/Polymer Solar Cells Based on Tetrapod-Shaped $\mathrm{CdSe}_{x} \mathrm{Te}_{1-x}$ Nanocrystals. Nanotechnology 2006, 17, 4041-4047.

(30) Zhong, H. Z.; Zhou, Y.; Yang, Y.; Yang, C. H.; Li, Y. F. Synthesis of Type II CdTe-CdSe Nanocrystal Heterostructured Multiple-Branched Rods and Their Photovoltaic Applications. J. Phys. Chem. C 2007, 111, 6538-6543.

(31) Cui, Y.; Banin, U.; Bjork, M. T.; Alivisatos, A. P. Electrical Transport through a Single Nanoscale Semiconductor Branch Point. Nano Lett. 2005, 5, 1519-1523.

(32) Nobile, C.; Ashby, P. D.; Schuck, P. J.; Fiore, A.; Mastria, R.; Cingolani, R; Manna, L.; Krahne, R. Probe Tips Functionalized with Colloidal Nanocrystal Tetrapods for High-Resolution Atomic Force Microscopy Imaging. Small 2008, 4, 2123-2126.

(33) Xie, R.; Zhong, X.; Basché, T. Synthesis, Characterization, and Spectroscopy of Type-II Core/Shell Semiconductor Nanocrystals with ZnTe Cores. Adv. Mater. 2005, 17, 2741-2745.

(34) Yu, K.; Zaman, B.; Romanova, S.; Wang, D.; Ripmeester, A. Sequential Synthesis of Type-II Colloidal CdTe/CdSe Core-Shell Nanocrystals. Small 2005, 1, 332-338.

(35) Chin, P. T. K.; de Mello Donegá, C.; van Bavel, S. S.; Meskers, S. C. J.; Sommerdijk, N. A. J. M.; Janssen, R. A. J. Highly Luminescent $\mathrm{CdTe} / \mathrm{CdSe}$ Colloidal Heteronanocrystals with Temperature-Dependent Emission Color. J. Am. Chem. Soc. 2007, 129, 14880-14886.

(36) Dooley, C. J.; Dimitrov, S. D.; Fiebig, T. Ultrafast Electron Transfer Dynamics in CdSe/CdTe Donor-Acceptor Nanorods. J. Phys. Chem. C 2008, 112, 12074-12076.

(37) de Mello Donegá, C. Synthesis and Properties of Colloidal Heteronanocrystals. Chem. Soc. Rev. 2011, 40, 1512-1546.

(38) de Mello Donegá, C. Formation of Nanoscale Spatially Indirect Excitons: Evolution of the Type-II Optical Character of CdTe/CdSe Heteronaocrystals. Phys. Rev. B 2010, 81, 165303-165323.

(39) Wang, C.; Kwon, K.-W.; Odlyzko, M. L.; Lee, B. H.; Shim, M. PbSe Nanocrystal/ $/ \mathrm{TiO}_{x}$ Heterostructured Films: A Simple Route to Nanoscale Heterointerfaces and Photocatalysis. J. Phys. Chem. C 2007, $111,11734-11741$.

(40) Chambers, S. A.; Williams, J. R.; Henderson, M. A.; Joly, A. G.; Varela, M.; Pennycook, S. J. Structure, Band Offsets and Photochemistry at Epitaxial $\alpha-\mathrm{Cr}_{2} \mathrm{O}_{3} / \alpha-\mathrm{Fe}_{2} \mathrm{O}_{3}$ Heterojunctions. Surf. Sci. 2005, 587, L197-L207.

(41) Das, K.; De, S. K. Optical Properties of the Type-II Core-Shell $\mathrm{TiO}_{2} @ \mathrm{CdS}$ Nanorods for Photovoltaic Applications. J. Phys. Chem. C 2009, 113, 3494-3501.

(42) Blumstengel, S.; Sadofev, S.; Henneberger, F. Electronic Coupling of Optical Excitations in Organic/Inorganic Semiconductor Hybrid Structures. New J. Phys. 2008, 10, 065010-065026.

(43) Kanai, Y.; Grossman, J. C. Role of Semiconducting and Metallic Tubes in P3HT/Carbon-Nanotube Photovoltaic Heterojunctions: Density Functional Theory Calculations. Nano Lett. 2008, 8, 908-912.

(44) Dorfs, D.; Salant, A.; Popov, I.; Banin, U. ZnSe Quantum Dots Within CdS Nanorods: A Seeded-Growth Type-II System. Small 2008, 4, 1319-1323.

(45) Fiore, A.; Mastria, R.; Lupo, M. G.; Lanzani, G.; Giannini, C.; Carlino, E.; Morello, G.; De Giorgi, M.; Li, Y.; Cingolani, R.; Manna, L. Tetrapod-Shaped Colloidal Nanocrystals of II-VI Semiconductors Prepared by Seeded Growth. J. Am. Chem. Soc. 2009, 131, 2274-2282.

(46) Talapin, D. V.; Koeppe, R.; Gotzinger, S.; Kornowski, A.; Lupton, J. M.; Rogach, A. L.; Benson, O.; Feldmann, J.; Weller, H. Highly Emissive Colloidal CdSe/CdS Heterostructures of Mixed Dimensionality. Nano Lett. 2003, 3, 1677-1681.

(47) Zhong, H.; Scholes, G. D. Shape Tuning of Type II CdTe-CdSe Colloidal Nanocrystal Heterostructures through Seeded Growth. J. Am. Chem. Soc. 2009, 131, 9170-9171.

(48) Xie, R. G.; Kolb, U.; Basché, T. Design and Synthesis of Colloidal Nanocrystal Heterostructures with Tetrapod Morphology. Small 2006, 2, 1454-1457. 
(49) Talapin, D. V.; Nelson, J. H.; Shevchenko, E. V.; Aloni, S.; Sadtler, B.; Alivisatos, A. P. Seeded Growth of Highly Luminescent $\mathrm{CdSe} / \mathrm{CdS}$ Nanoheterostructures with Rod and Tetrapod Morphologies. Nano Lett. 2007, 7, 2951-2959.

(50) Milliron, D. J.; Hughes, S. M.; Cui, Y.; Manna, L.; Li, J.; Wang, L.-W.; Alivisatos, A. P. Colloidal Nanocrystal Heterostructures with Linear and Branched Topology. Nature 2004, 430, 190-195.

(51) Lee, H.; Yoon, S. W.; Ahn, J. P.; Suh, Y. D.; Lee, J. S.; Lim, H.; Kim, D. Synthesis of Type-II CdTe/CdSe Heterostructure Tetrapod Nanocrystals for PV Applications. Sol. Energy Mater. Sol. Cells 2009, 93, 779-782.

(52) Li, Y.; Mastria, R.; Li, K.; Fiore, A.; Wang, Y.; Cingolani, R.; Manna, L.; Gigli, G. Improved Photovoltaic Performance of Bilayer Heterojunction Photovoltaic Cells by Triplet Materials and TetrapodShaped Colloidal Nanocrystals Doping. Appl. Phys. Lett. 2009, 95, 043101-043103.

(53) Li, Y.; Mastria, R.; Fiore, A.; Nobile, C.; Yin, L.; Biasiucci, M.; Cheng, G.; Cucolo, A. M.; Cingolani, R.; Manna, L.; Gigli, G. Improved Photovoltaic Performance of Heterostructured Tetrapod-Shaped CdSe/CdTe Nanocrystals Using C60 Interlayer. Adv. Mater. 2009, 21, 4461-4466.

(54) Peng, P.; Milliron, D. J.; Hughes, S. M.; Johnson, J. C.; Alivisatos, A. P.; Saykally, R. J. Femtosecond Spectroscopy of Carrier Relaxation Dynamics in Type II CdSe/CdTe Tetrapod Heteronanostructures. Nano Lett. 2005, 5, 1809-1813.

(55) Tarì, D.; De Giorgi, M.; Della Sala, F.; Carbone, L.; Krahne, R.; Manna, L.; Cingolani, R.; Kudera, S.; Parak, W. J. Optical Properties of Tetrapod-Shaped CdTe Nanocrystals. Appl. Phys. Lett. 2005, 87, 224101-224103.

(56) Chin, P. T. K.; de Mello Donegá, C.; van Bavel, S. S.; Meskers, S. C. J.; Sommerdijk, N. A. J. M.; Janssen, R. A. J. Highly Luminescent $\mathrm{CdTe} / \mathrm{CdSe}$ Colloidal Heteronanocrystals with Temperature-Dependent Emission Color. J. Am. Chem. Soc. 2007, 129, 14880-14886.

(57) Balet, L. P.; Ivanov, S. A.; Piryatinski, A.; Achermann, M.; Klimov, V. I. Inverted Core/Shell Nanocrystals Continuously Tunable between Type-I and Type-II Localization Regimes. Nano Lett. 2004, 4, 1485-1488.

(58) Jones, M.; Kumar, S.; Lo, S. S.; Scholes, G. D. Exciton Trapping and Recombination in Type II CdSe/CdTe Nanorod Heterostructures. J. Phys. Chem. C 2008, 112, 5423-5431.

(59) Kumar, S.; Jones, M.; Lo, S. S.; Scholes, G. D. Nanorod Heterostructures Showing Photoinduced Charge Separation. Small 2007, 3, 1633-1639.

(60) He, J.; Lo, S. S.; Kim, J.; Scholes, G. D. Control of Exciton Spin Relaxation by Electron-Hole Decoupling in Type-II Nanocrystal Heterostructures. Nano Lett. 2008, 8, 4007-4013.

(61) Wang, C. H.; Chen, T. T.; Tan, K. W; Chen, Y. F.; Cheng, C. T.; Chou, P. T. Photoluminescence Properties of CdTe/CdSe Core-Shell Type-II Quantum Dots. J. App. Phys. 2006, 99, 123521-123524.

(62) Binder, R.; Galbraith, I.; Koch, S. W. Theory of Band-Edge Optical Nonlinearities in Type-I and Type-II Quantum-Well Structures. Phys. Rev. B 1991, 44, 3031-3042.

(63) Morello, G.; Tarì, D.; Carbone, L.; Manna, L.; Cingolani, R.; De Giorgi, M. Radiative Recombination Dynamics in Tetrapod-Shaped CdTe Nanocrystals: Evidence for a Photoinduced Screening of the Internal Electric Field. Appl. Phys. Lett. 2008, 92, 191905-191907.

(64) Steiner, D.; Dorfs, D.; Banin, U.; Della Sala, F.; Manna, L.; Millo, O. Determination of Band Offsets in Heterostructured Colloidal Nanorods Using Scanning Tunneling Spectroscopy. Nano Lett. 2008, 8, 2954-2958.

(65) Valerini, D.; Cretì, A.; Lomascolo, M.; Manna, L.; Cingolani, R.; Anni, M. Temperature Dependence of the Photoluminescence Properties of Colloidal CdSe/ZnS Core/Shell Quantum Dots Embedded in a Polystyrene Matrix. Phys. Rev. B 2005, 71, 235409-235415.

(66) Morello, G.; De Giorgi, M.; Kudera, S.; Manna, L.; Cingolani, R; Anni, M. Temperature and Size Dependence of Nonradiative Relaxation and Exciton-Phonon Coupling in Colloidal CdTe Quantum Dots. J. Phys. Chem. C 2007, 111, 5846-5849.
(67) Harrison, P. Quantum Wells, Wires and Dots: Theoretical and Computational Physics of Semiconductor Nanostructures; Wiley: New York, 2005.

(68) Cretì, A.; Anni, M.; Rossi, M. Z.; Lanzani, G.; Leo, G.; Della Sala, F.; Manna, L.; Lomascolo, M. Ultrafast Carrier Dynamics in Core and Core/Shell CdSe Quantum Rods: Role of the Surface and Interface Defects. Phys. Rev. B 2005, 72, 125346-125355.

(69) Morello, G.; Della Sala, F.; Carbone, L.; Manna, L.; Maruccio, G.; Cingolani, R.; De Giorgi, M. Intrinsic Optical Nonlinearity in Colloidal Seeded Grown CdSe/CdS Nanostructures: Photoinduced Screening of the Internal Electric Field. Phys. Rev. B 2008, 78, 195313195321.

(70) Lupo, M. G.; Della Sala, F.; Carbone, L.; Zavelani-Rossi, M.; Fiore, A.; Luer, L.; Polli, D.; Cingolani, R.; Manna, L.; Lanzani, G. Ultrafast Electron-Hole Dynamics in Core/Shell CdSe/CdS Dot/Rod Nanocrystals. Nano Lett. 2008, 8, 4582-4587.

(71) Sitt, A.; Della Sala, F.; Menagen, G.; Banin, U. Multiexciton Engineering in Seeded Core/Shell Nanorods: Transfer from Type-I to Quasi-type-II Regimes. Nano Lett. 2009, 9, 3470-3476.

(72) Li, T. L.; Kuhn, K. J. Effects of Mass Discontinuity on the Numerical Solutions to Quantum Wells Using the Effective Mass Equation. J. Comput. Phys. 1994, 110, 292-300.

(73) Franchini, I. R.; Cola, A.; Rizzo, A.; Mastria, R.; Persano, A.; Krahne, R.; Genovese, A.; Falqui, A.; Baranov, D.; Gigli, G.; Manna, L. Phototransport in Networks of Tetrapod-Shaped Colloidal Semiconductor Nanocrystals. Nanoscale 2010, 2, 2171-2179.

(74) Gross, D.; Susha, A. S.; Klar, T. A.; Da Como, E.; Rogach, A. L.; Feldmann, J. Charge Separation in Type II Tunneling Structures of Close-packed CdTe and CdSe Nanocrystals. Nano Lett. 2008, 8, 14821485.

(75) Lo, S. S.; Mirkovic, T.; Chuang, C. H.; Burda, C.; Scholes, G. D. Emergent Properties Resulting from Type-II Band Alignment in Semiconductor Nanoheterostructures. Adv. Mater. 2011, 23, 180-197.

(76) Kagan, C. R; Murray, C. B.; Bawendi, M. Long-Range Resonance Transfer of Electronic Excitation in Close-Packed CdSe Quantum-Dot Solids. Phys. Rev. B 1996, 54, 8633-8643. 